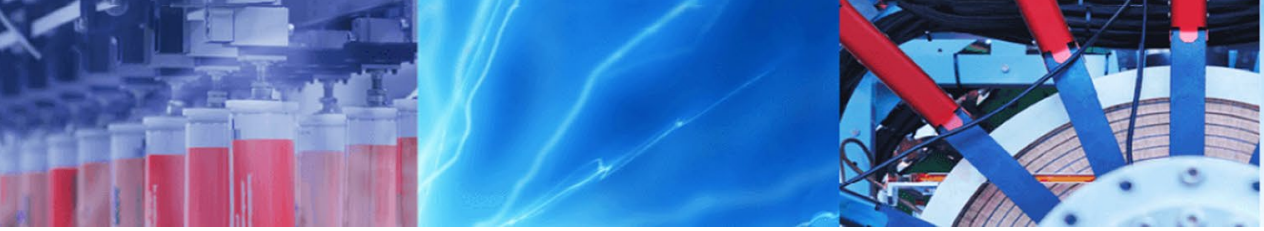

Research Article

\title{
Optimal tuning of FOPID controller based on PSO algorithm with reference model for a single conical tank system
}

\author{
R. Rajesh ${ }^{1}$ iD \\ (c) Springer Nature Switzerland AG 2019
}

\begin{abstract}
The fractional order proportional integral derivative controller (FOPID) replaces the conventional PID controller for its immense merits such as its simple structure, better set point tracking, high disturbance rejection, higher capability of handling model uncertainties in nonlinear and real time applications. This paper addresses the effectiveness of the FOPID controller on a level control of single conical system in real time. It is one of the classical nonlinear control problem and its shape not only contributes the proper mixing of liquids and also guarantee to drainage of solid wastes. This study, a dual loop controller is proposed and constructed with a master process and slave process for a level control of a single conical tank system. In the slave process, the output response obtained by the traditional PID controller combined with the conical tank reference model and compared with the output response of the master process. The obtained error signal is used to dynamically correct the parameters of the FOPID controller in the master process. The tuning of FOPID controller is a challenging task due to its presence of extra parameters and it efficiently carried out by particle swarm optimization (PSO) algorithm. The obtained result reveals that the Proposed PSO optimized FOPID controller with reference model demonstrated the better set point tracking, smooth controller response than other conventional integer order controllers.
\end{abstract}

Keywords FOPID controller · Single conical tank system · Reference model · PSO algorithm

\section{Introduction}

Fractional order calculus is a famous mathematical area with over 300 years of antiquity that generalizes a conventional integer calculus into arbitrary orders. In the initial theory of fractional order derivative was developed in the seventeenth epoch assumption between L'Hospital and Leibniz [1]. Mostly, real time problems can be described, defined, modeled and controlled more precisely in fractional order methods than the integral order methods. Several engineering fields and science applications utilize fractional calculus due to well theoretical explanation and development of the computing area in the last two decades. Subsequently, new design tools makes fractionalorder integro-differential equations more effortlessly.
Moreover, the applications of fractional-order differential equations has got in different prospects in control systems. Accordingly, various research work has been completed in the fractional order control (FOC) in the last decades due to its extra flexibility to meet control applications more specifically.

Conventional PID is the utmost typical controller employed in the process industries in last six decades, because of simplicity, ease of implementation, availability of many tuning methodologies and minimum knowledge of the process enough to control. However, there is no guarantee of dynamic responses, structural complexity, nonlinearities, and large time delays. The uncertainties in some realistically constrained cases in modern industries is makes increasing the attention for an improvement of

R. Rajesh, ra.rajeshkrishna@gmail.com | 'Department of Instrumentation and Control Engineering, Sri Krishna College of Technology, Coimbatore, India.

SN Applied Sciences (2019) 1:758 | https://doi.org/10.1007/s42452-019-0754-3 
PID controller. Podlubny et al. [2] has stretched integer order PID controller theory to fractional order PID controller and suggested in fractional order formats of PI and PID controller i.e. $P I^{\lambda}$ and $P I^{\lambda} D^{\mu}$. These controllers have an integrator order $\lambda$ and differentiator order $\mu$ and this two extra parameter provides an added degree of freedom in the performance of controller that makes FOPID controller performance better than conventional PID controller. Then Podlubny's works related to fractional order theory and fractional order controller has given lot of new ideas to researches and industrial real time application. Some of the very important literatures are: Roy et al. [3] developed adaptive fractional order PI controller for a variable quadruple tank process with feed forward controller and compared with decentralized $\mathrm{PI}$ and sliding mode controller. Padula et al. [12] proved advantages of FOPID controller than other integer order controller with large number of experiment under set point following and load disturbance rejection. This paper also minimize the integrated absolute error subject to a constraint to the maximum sensitivity. Ranganayakula et al. [4] studied the different tuning rules for a FOPI and FOPID controllers and validated using different stable first order time delay processes. Sharma et al. [5] developed fuzzy FOPID controller for a robotic manipulator. This paper shows advantages of FOPID controller under trajectory tracking, model uncertainty, disturbance rejection and noise rejection compared to the other integer order controller. Azarmi et al. [6] demonstrated and proved FOPID controller for a CE 150 type laboratory helicopter model is better than PID and FOPI controller under change in disturbance. Moreover, the flexibility of handling uncertainties, robustness, sinking undesired oscillations and fast change of control signal makes requirement of fractional order controller concepts into many advanced control strategies such as phase lead lag compensator $[7,8]$, sliding mode control based FOC $[9,10]$, quadratic regulator based FOC [11, 12], smith predictor based FOC [13], internal model based FOC $[14,15], H_{\infty}$ norm based FOC [16, 17], set-point weighted FOC [18], FOC with pre-filter [19] and Loop shaping method [20]. From the above literature clearly shows FOPID controller is better than other integer order controller. Also, currently many researchers chosen FOPID controller due to extra parameters makes system more robust and effective for different applications.

The fine tuning of fractional order PID control is challenging compared to conventional PID control due to the existence of extra two parameters and to meet some special constraints like gain margin, phase margin, gain crossover frequency, and sensitivity conditions. Nevertheless, evident from a literature shows that the development of the meta-heuristic methods are created the tuning of constraints very ease [21-23] in last few years such as genetic algorithm [24, 25], Big bang big crunch algorithm [26], particle swarm optimization [27], bacterial foraging optimization algorithm $[22,23,28]$, artificial bee colony algorithm $[29,30]$, stochastic multi- parameters divergence optimization [31], multi objective optimization design [32], cuckoo search algorithm [33, 34], bacterial foraging chemotaxis gravitational search algorithm [35], differential evolution [36], chaotic ant swarm optimization [37], gases brownian motion optimization [38], bat algorithm [39], tabu search algorithm [40] than analytical approach.

Even though development of many natural inspired algorithms, PSO has got some constant place among researchers due to its simplicity, simple calculation, lots of freedom given to modify the structure of algorithm and a substitute solution to the non-linear complex optimization problem. Its base idea was stimulated from the social activities of animals such as fish schooling, bird clustering, etc. PSO depends on the natural activity of communication between the teams and to share individual information when a team of birds or insects searching food or throughout migration. All the birds don't know wherever the best position, however from the social behavior, if anyone of the members within the group can find the desired path to travel and therefore remainder of the particle track quickly. In this article, to optimize the FOPID controller parameters PSO algorithm is concentrated.

As far as design for tuning of PID and FOPID controller, performance index such as integral square error (ISE), integral absolute error (IAE), and integral time absolute error (ITAE) only used in an objective function. IAE and ISE produce comparatively minor overshoot but a lengthy settling time. Though ITAE surpassing this drawback, but produces problem in the stability margin. In this article includes time domains performance criteria such as settling time, rise time, and peak overshoot with weighting values in the objective function to improve the optimization performance. Recently many researchers are giving importance for tuning of PID controller with reference model and this idea was originate from the concept of model reference adaptive control. A reference model is designed based on the expected output from the plant model [41]. Recently, many researchers given importance to tuning the controller with reference model. Barbosa et al. [42] and Li et al. [43] utilized Bode's ideal transfer function for tuning PID controller. Neural network based reference model is proposed for positioning control system by [41] and [44]. Wang et al. [45] developed reference model based sliding mode control for helicopter system. Jeng [46] addressed different specifications for a reference model and analyses the control strategy with reference model for a different stable and unstable plants. In this article, time domain criteria such as settling time and peak overshoot values are utilized for conical system reference model implementation. In the proposed dual loop 
structure, closed loop reference model with PID controller setup is known as slave process and plant model with FOPID controller is known as a master process. The difference between the response from the reference model and plant response is considered as an error which is used as an objective function with some time domain specifications for tuning of FOPID controller parameters using PSO algorithm. This difference of error value improves the efficiency of the controller to perform control in the plant.

The proposed dual loop control structure is validated in real time using highly nonlinear single conical tank system. It is one of the classical nonlinear control problem whose objective is to control the level of the tank. This shape not only contributes the proper mixing of liquids and also guarantee to drainage of solid wastes [47]. This type structure widely used in food factories, petroleum industries and chemical industries. So controlling of conical tank is important and challenging task in process control industry. If the level of the tank increases, then overflow of the valuable liquid. Similarly decreases in the level produces bad significances in the sequential operation. Hence dedicated and efficient control algorithm is required for efficient operation. Vijayalakshmi et al. [48] developed linear parameter varying model and then controlled using adaptive PI controller. Ramanathan et al. [47] presented reinforcement learning technique based on Q-learning algorithm for a conical tank level control. Ravi et al. [49] demonstrated regime-based multi-model adaptive control strategy for decoupling-based decentralized PI controller for a conical tank system. Tamilselven et al. [50] estimated the plant parameters and control using online kalman filter based fuzzy logic controller. Similarly, adaptive passivity based adaptive control algorithm with Taylor polynomial approximation for a level regulation of a conical tank system is developed by [51]. Lot of control algorithm is proposed for control of conical tank system but still, it is an open issue in current and future. In this article proposed FOPID controller with reference model is proposed for level control of a single conical tank system.

The major contributions of this article are,

1. Better controller performance and smooth control signal is achieved using dual loop FOPID control structure with reference model for a single conical tank system.

2. The difference between the reference model and plant model, dynamically adjust the FOPID controller parameter by using PSO algorithm with multi-objective function.

This paper is prepared as, a detailed review of fractional order calculus and FOPID controller is described in Sect. 2. Section 3 comprises the detail description of single conical tank system and first order plus dead time approximation. Overview of PSO is explained in Sect. 4. A detail block diagram arrangement of the proposed technique and reference model formation of a single conical tank system with multi-objective function is given in Sect. 5. A practical result of the FOPID controller based PSO algorithm with reference model for a single conical tank system is described in Sect. 6. Lastly Sect. 7 concludes article.

\section{Review of fractional calculus and FOPID controller}

The first study of fractional calculus was described by Liouville (1832), who was principally involved by deep components of Laplace and Fourier. Later M. Axtell discussed and recommended an extensive usage of fractional calculus in control system in 1990, first time. The detailed review of fractional order calculus and FOC is available in [52-54].

\subsection{Fractional order calculus and approximation}

This is the generalized illustration of the differentiation and integration for non-integer order fundamental operator ${ }_{\beta} D_{t}^{\chi}$ where $\chi$ and $t$ are limits of an operator. The integrodifferential operator in continuous domain is defined as

${ }_{\beta} D_{t}^{\chi}= \begin{cases}\frac{\mathrm{d}^{\chi}}{d t \chi} & \Re(\chi)>0 \\ 1 & \mathfrak{R}(\chi)>0 \\ \int_{a}^{t}(\mathrm{~d} \tau)^{-\chi} & \Re(\chi)>0\end{cases}$

where $\chi \in \mathbb{R}$ but $\chi$ could be a complex number and $\beta$, $t$ are limits of an operator. Some definitions available for fractional order calculus in literature but the commonly used are as follows.

Definition 1 Grunwald-Letnikov is defined as,

${ }_{\beta} D_{t}^{\chi} f(t)=\lim _{h \rightarrow 0} \frac{1}{g^{\chi}} \sum_{q=0}^{\left[\frac{t-\beta}{g}\right]}(-1)^{q}\left(\begin{array}{l}b \\ q\end{array}\right) f(t-q g)$

where, $\left[\frac{t-\beta}{g}\right]$ is the integer part and $b$ is the integer which fulfills the stipulation $b-1 \leq \chi<b$. 
$\left(\begin{array}{l}b \\ q\end{array}\right)=\frac{\Gamma(b+1)}{\Gamma(q+1) \Gamma(p-q+1)}$

$\Gamma(\cdot)$ is the gamma function.

Definition 2 Riemann-Lioville is defined by,

${ }_{\beta} D_{t}^{\chi} f(t)=\frac{1}{\Gamma(b-\chi)}\left(\frac{d}{d t}\right)^{b} \int_{\beta}^{t} \frac{f(\tau)}{(t-\tau)^{\chi-b+1}} d \tau$

Definition 3 Caputo fractional is defined by,

${ }_{\beta} D_{t}^{\chi} f(t)=\frac{1}{\Gamma(b-\chi)}\left(\frac{d}{d t}\right)^{b} \int_{\beta}^{t} \frac{f^{b}(\tau)}{(t-\tau)^{\chi-b+1}} d \tau$

Fractional order differential equation is not a simpler in numerical simulation like a standard differential equation. Therefore, Laplace transforms tool is frequently utilized by engineers, especially control engineering applications.

The Laplace transform of Riemann-Lioville definition is as follows $[55,56]$

$L_{a}\left\{{ }_{0} D_{t}^{\chi} f(t) ; s\right\}=s^{\chi}(L f(t))-\sum_{l=0}^{(n-1)} s^{l}\left[{ }_{0} D_{t}^{(\chi-l-1)} f(t)\right] t=0$

Laplace transform of Caputo's representation is as, [57]

$L\left\{{ }_{0} D_{t}^{\chi} f(t) ; s\right\}=s^{\chi}(L f(t))-\sum_{l=0}^{(n-1)} s^{(\chi-I-1)} f^{\prime}(0)$

where $s=j \omega$ represents the Laplace operator. For null initial conditions, Laplace transform of Riemann-Liouville fractional derivative and Caputo's fractional derivative are equivalent, it's given in $(8)[57,58]$

$L\left\{{ }_{0} D_{t}^{\chi} f(t)\right\}=s^{\chi}(L f(t))$

Hence, the fractional integer operator $\beta$ embodied by the transfer function $L f(t)=F(s)=1 / s^{x}$ in the frequency domain.

\subsection{Integer-order approximation of fractional derivative}

The fractional differential equation is not simply like an ordinary differential equation and it is very tough to develop in the time domain. This can be addressed by approximate the fractional operator using standard integer-order operator. In this article, fractional order term $S^{x}$ is approximated using Oustaloup's filter $[59,60]$ method. $s^{\chi} \approx K \prod_{b=-B}^{B} \frac{1+\left(s / \omega_{z, b}\right)}{1+\left(s / \omega_{p, b}\right)}, \chi>0$

$2 B+1$ is the number of poles and zeros selected earlier. $K$ is gain adjusted so that the approximation shall have a unit gain at $1 \mathrm{rad} / \mathrm{s} . \omega_{z, b}$ and $\omega_{p, b}$ are,

$\omega_{z, b}=\omega_{a}\left(\frac{\omega_{g}}{\omega_{a}}\right)^{\frac{b+B+(1-x) / 2}{2 B+1}}$

$\omega_{p, b}=\omega_{a}\left(\frac{\omega_{g}}{\omega_{a}}\right)^{\frac{b+B+(1+x) / 2}{2 B+1}}$

In (10) and (11), $\omega_{a}$ and $\omega_{g}$ are limits of frequency approximation. Generally $\omega_{a} \omega_{g}$ equal to one and thus $K=\omega_{g}^{\chi}$. For $|\chi|>1$, the approximation becomes unsatisfactory those cases fractional power of $\mathrm{s}$ is split like,

$s^{\chi}=s^{b} s^{\delta}, \chi=b+\delta, b \in \mathrm{Z}, \delta \in[0,1]$

\subsection{Fractional order proportional integral derivative controller}

Fractional order PID controller $\left(P I^{\lambda} D^{\mu}\right)$ is the continuation of conventional PID control and $\lambda, \mu \in \mathbb{R}$. From the literature can also observe that FOPID controller enhance the system performance, less sensitive to change of parameters and attain property of iso-damping very easily. General form of FOPID controller is defined as,

$C(s)=\frac{U(s)}{E(s)}=K_{p}+\frac{K_{i}}{s^{\lambda}}+K_{d} s^{\mu},(\lambda, \mu \geq 0)$

where $C(s)$ is the transfer function model of FOPID controller, $\mathrm{E}(\mathrm{S})$ is an error signal and $\mathrm{U}(\mathrm{s})$ is controller's output and block diagram of FOPID controller is presented in Fig. 1. The control signal $\mathrm{u}(\mathrm{t})$ is stated in time domain as,

$u(t)=K_{p}+K_{i} D^{-\lambda} e(t)+K_{d} D^{\mu} e(t)$

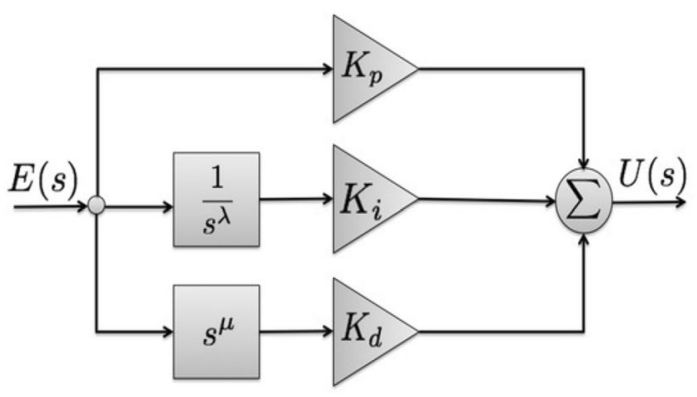

Fig. 1 Block diagram FOPID controller 


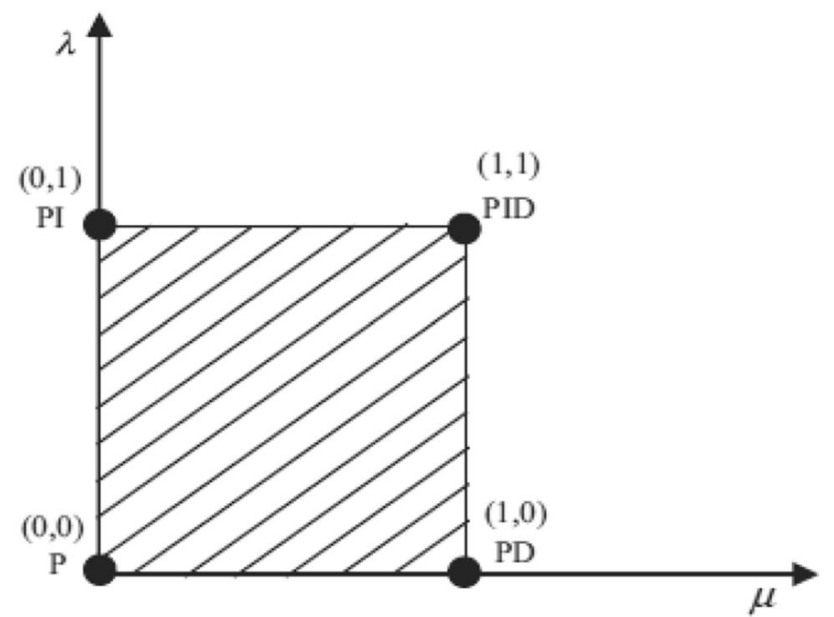

Fig. 2 FOPID controller convergence from point to point representation

By choosing $\lambda=1$ and $\mu=1$, a conventional PID controller is develop again. Similarly other combination is represented in the Fig. 2.

\section{Description of a single conical tank system}

Adjusting the level of a conical tank system is a very interesting task, owing to their variations in the control area, uncertainty, time varying parameters and dead time on input and output variables. Nonlinear systems like conical tanks used in wide choice of applications such as gas plants, food industries, petrochemical plants because of merits like better disposal of solids and easy mixing. In this section real time setup, mathematical modeling and identification of conical tank system is described.

\subsection{Real time setup and technical specifications of single conical tank system}

Real time conical tank system consists of one input (inflow) and one output (level of the tank) as shown in Fig. 3 and experimental setup is illustrated in Fig. 4. The inflow is taken from the reservoir tank through the centrifugal pump with single phase motor. The inlet pipe has rotometer, flow transmitter, air-to-open type pneumatic control valve and a hand valve to control the flow rate and this same setup of equipment available in outlet pipe side as well. The level in the tank is directly proportional to the pressure created by the liquid in it and this level inside the tank is measured with the differential pressure transducer which is calibrated for $0-450 \mathrm{~mm}$ and is converted to an output range of 4-20 mA. This converted output current from differential pressure transmitter (DPT) is

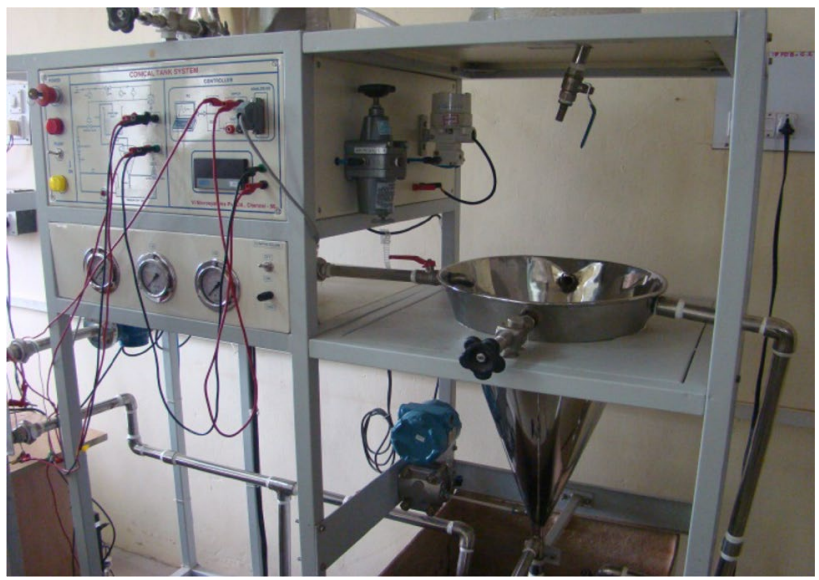

Fig. 3 Real time setup of a single conical tank system

passed through I/V converter to convert into standard voltage range of $1-5 \mathrm{~V}$ which is acquired by DAQ module of National Instruments Ltd. The detailed technical specification of single conical tank system is shown in Table 1.

\subsection{Modeling of a single conical tank system}

The objective is to control a level of the tank, which can be achieved by controlling the input flow of the conical tank by using a valve at an inlet. At steady state, both the inflow $\left(F_{i}\right)$ and outflow $\left(F_{o}\right)$ rates remain the same. At each height of the conical tank, the radius will vary due to the nonlinear nature which is due to the shape of the tank. The difference between the inflow and the outflow rate will be based upon the cross section area of the tank and level of the tank with respect to time. The flow and the level of the tank can be regulated by properly modeling the tank.

The area of the conical tank $(A)$ is given by

$A=\pi r^{2}$

At any level (h),

$\tan \theta=\frac{r}{h}=\frac{R}{H}$

where $\theta$ is the angle difference which relates the current height of fluid to the total height of the tank (Degrees), $R$ is the maximum radius of the tank and $\mathrm{H}$ is the maximum height of the tank.

$r=R \frac{h}{H}$

Therefore cross sectional area of the $\operatorname{tank}(\mathrm{A})$ is

$A=\frac{\pi R^{2} h^{2}}{H^{2}}$

According to law of conservation of mass 
Fig. 4 Experimental setup of a single conical tank system

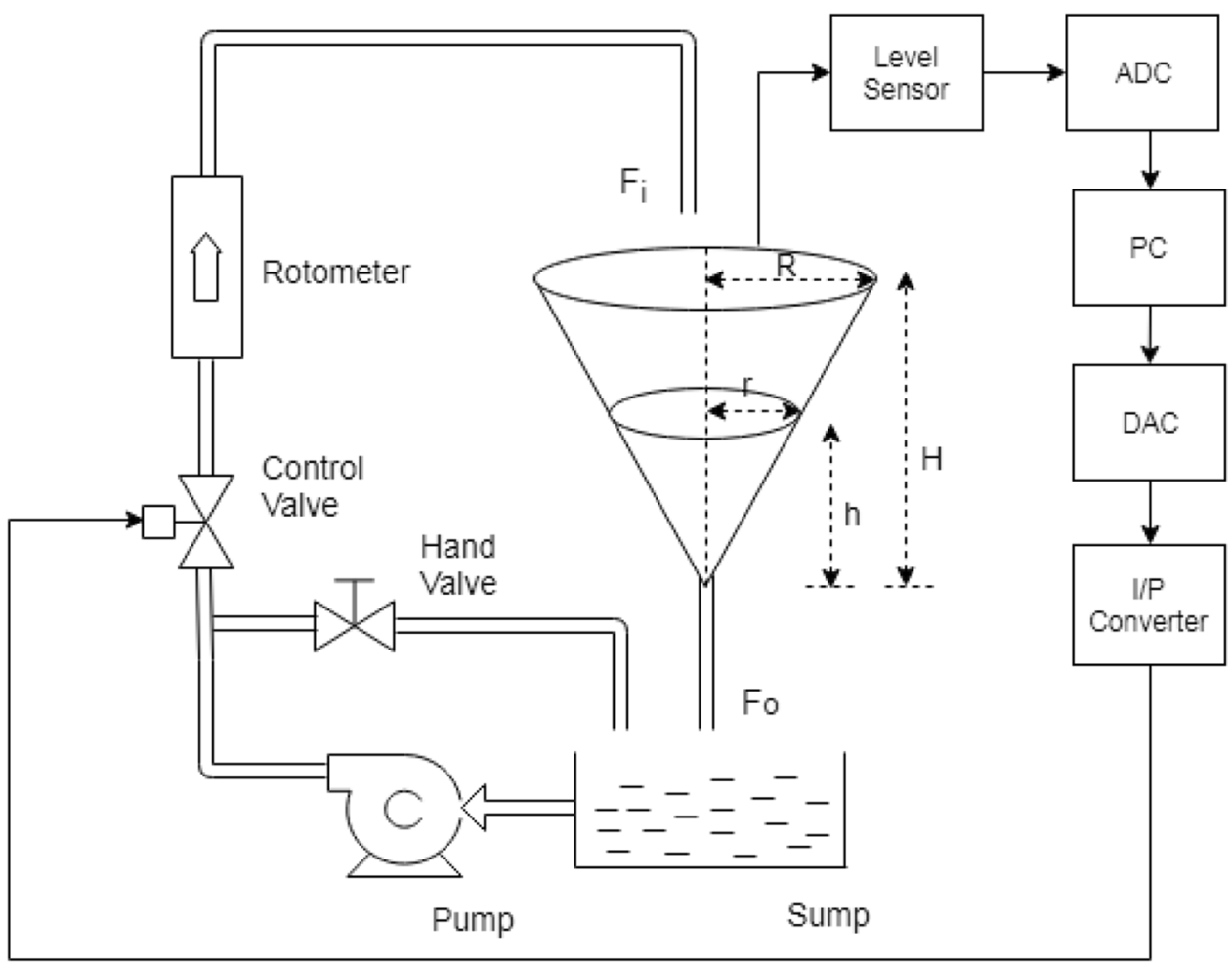

Table 1 Technical specifications of a single conical tank system

\begin{tabular}{|c|c|}
\hline Part name & Technical specifications \\
\hline Conical tank & $\begin{array}{l}\text { Stainless steel body } \\
\text { Top radius (R): } 240 \mathrm{~mm} \\
\text { Bottom radius (r): } 8 \mathrm{~mm} \\
\text { Height }(\mathrm{H}): 50 \mathrm{~cm} \\
\text { Inflow: } 0-1000 \mathrm{lph}\end{array}$ \\
\hline Differential pressure transmitter & $\begin{array}{l}\text { Capacitive type } \\
\text { Range : } 2.5-250 \mathrm{mbar} \\
\text { Output : } 4-20 \mathrm{~mA}\end{array}$ \\
\hline Pump type & $\begin{array}{l}\text { Centrifugal } 0.5 \mathrm{HP} \\
\text { Single phase } \mathrm{AC} \text { motor }\end{array}$ \\
\hline Control valve & $\begin{array}{l}\text { Size : } 1 / 4^{\prime \prime} \\
\text { Equal percentage valve } \\
\text { Air-open type } \\
\text { Input : } 3-15 \text { psi }\end{array}$ \\
\hline Rotameter & Range: 0-18 lpm \\
\hline Air regulator & $\begin{array}{l}\text { Size: } 1 / 4^{\prime \prime} \text { BSP } \\
\text { Range: } 0-2.2 \text { bar }\end{array}$ \\
\hline I/P converter & $\begin{array}{l}\text { Input: } 4-20 \mathrm{~mA} \\
\text { Output: } 0.2-1 \mathrm{bar}\end{array}$ \\
\hline Pressure gauge & Range: $0-30 \mathrm{psi}$ \\
\hline Compressor & 20 psi \\
\hline
\end{tabular}

Inflow rate $\left(F_{i}\right)$ - outflow rate $\left(F_{o}\right)=$ Accumulation in the tank

$F_{i}-F_{0}=A \frac{d h}{d t}$
Then outflow $\left(F_{o}\right)$ can be written as,

$F_{0}=C \sqrt{h}$

where, $C$ is the valve discharge coefficient of the outlet valve.

On solving (20) in (19),

$F_{i}-C \sqrt{h}=A \frac{d h}{d t}$

$\frac{d h}{d t}=\frac{F_{i}-C \sqrt{h}}{A}$

On solving (18) in (22)

$\frac{d h}{d t}=\frac{F_{i} H^{2}}{\pi(R h)^{2}}-\frac{C \sqrt{h} H^{2}}{\pi(R h)^{2}}$

Then rate of change of height $(d h / d t)$ can be written as,

$\frac{d h}{d t}=\alpha F_{i} h^{-2}-\beta h^{(-3 / 2)}$

where,

$\alpha=\frac{1}{\pi}\left(\frac{H}{R}\right)^{2}$

$\beta=\alpha C$ 


\subsection{FOPDT approximation of a single conical tank system}

The first order plus dead time (FOPDT) model is not alone delivers the simple description of a process, however is identified to capture properly well dynamics of the numerous applications in process control industry. Very important in the representation of dead time delay in transfer function is to accurate control in real-time and most of PID tuning techniques are derived from FOPDT model. In general FOPDT model represented as,

$G_{m}=\frac{K e^{-s \tau}}{1+s T}$

where $K$ is process gain, $\tau$ is dead time and $T$ is time constant of process.

FOPDT approximation is one of the important research areas among process control and chemical engineers in more than 70 years. Ziegler and Nichols [61] and Cheng and Hung [62] founded the parameters of FOPDT model by creating the tangent on the open loop step response at the point of inflection. These crossing of the tangent with the time axis offers the time delay but in practice, location of the inflection point is difficult, may not be precise and computationally complex. Prabhu and Chidambaram [63] observed the parameters of the FOPDT model from the process reaction curve. Sundaresan and Krishnaswamy [64] founded a method based on the two time instants of the process reaction curve for predicting the dead time and time constant of FOPDT model. This technique consists of the estimation of two time instants $t_{1}$ and $t_{2}$ when the open loop reaches $35.3 \%$ and $85.3 \%$ of its last steady state value respectively. This method is very simple and effective compared to other techniques in the literature. The open loop response of single conical tank system as shown in Fig. 5. By using estimated $t_{1}$ and $t_{2}$ values dead time calculated as follows:

$\tau=0.67\left(t_{1}-t_{2}\right)$

Dead time obtained from the open loop response is around $178 \mathrm{~s}$ and thus the transfer function representation for a single conical tank system is given in (29).

$G_{p}=\frac{1.678 e^{-178 s}}{730.67 s+1}$

\section{Particle swarm optimization}

This section already explain in [41], and is repeated in this article for a sake of completeness. PSO is a unique population based stochastic search algorithm proposed by Kennedy and Eberhart [65]. The original idea of PSO algorithm is inspired from a model of the communal performance of animals such as fish schooling and bird gathering. This algorithm is based on communication of individual knowledge and natural learning when birds or insects hunt for food or migration in the search space. Popularity of PSO in the last decades due to its simple structure and only few parameters needed to adjust the optimization of any kind of problems.

In PSO, each possible result of the problem is considered as a bird or particle represented as $i \in(1,2, \ldots, m)$ and $m>1$. The set of particle is in a colony $(m)$ is flown in the $D$ dimensional search
Fig. 5 Real time open loop response of a single conical tank system

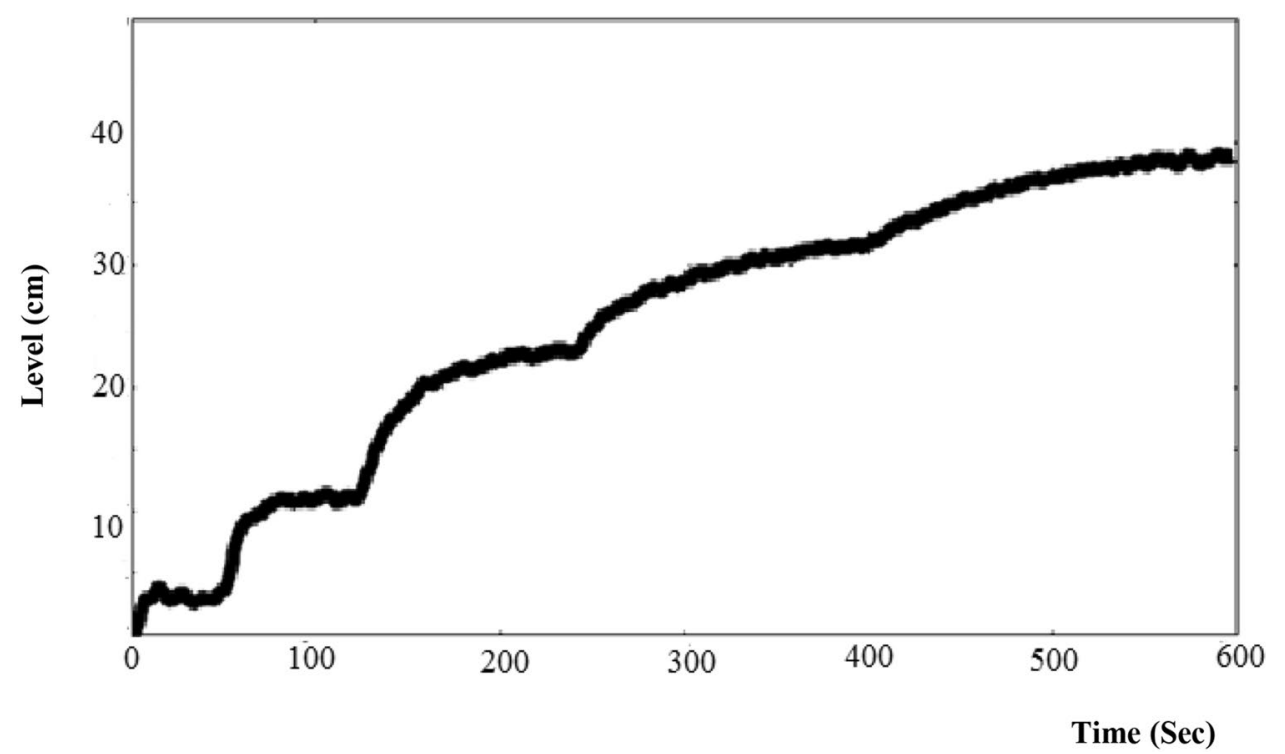

SN Applied Sciences 
planetary. This location of every particle is represented as $X_{i}(t)=\left(x_{i 1}(t), x_{i 2}(t), \ldots, x_{i D}(t)\right)$. The $\mathrm{D}$ dimensional space is created by $X_{i}(t) \in\left[I_{d}, u_{d}\right]$ where $I_{d}$ and $u_{d}$ are the limits. The velocity vector also represents like a position vector $V_{i}(t)=\left(v_{i 1}(t), v_{i 2}(t), \ldots, v_{i D}(t)\right)$. Each particle tracks the personal best particle position from its own experience and particle fitness value calculated from objective function, which is represented as $P_{b_{i}}(t)=\left(p_{b_{i} 1}(t), p_{b_{i} 2}(t), \ldots, p_{b_{i} D}(t)\right)$. An optimal value among the $P_{b_{i}}$ is called $G_{b}$ and it is represented as $G_{b}(t)=\left(g_{b 1}(t), g_{b 2}(t), \ldots, g_{b D}(t)\right)$. A PSO algorithm is worked based on (30-33). performance of the particle relative to the past performance or distance at which a particle is situated from the best solution $P_{b_{i}}$ found by its own. The third term $c_{2} \times \operatorname{rand}_{2}(t) \times\left(g_{b}(t)-x_{i}(t)\right)$ is termed as a social component that measures the performance of the particle relative to a bunch of particle or neighbors and the best solution determined by its neighbor is $G_{\text {best }}$. There is no control mechanism for velocity in the first version of PSO and makes the inefficient behavior of the algorithm. Later Shi and Eberhart [66] address this problem and produce inertial weight $(w)$ component in velocity

$v_{i}(t+1)=v_{i}(t)+c_{1} \times \operatorname{rand}_{1}(t) \times\left(p_{b_{i}}(t)-x_{i}(t)\right)+c_{2} \times \operatorname{rand}_{2}(t) \times\left(g_{b}(t)-x_{i}(t)\right)$

$x_{i}(t+1)=x_{i}(t)+v_{i}(t+1)$

The finest local location of every particle is updated as follows:

$p_{b_{i}}(t+1)= \begin{cases}p_{b_{i}}(t) & \text { if } f\left(\mathrm{x}_{\mathrm{i}}(t+1)\right)>p_{b_{i}}(t) \\ x_{i}(t+1) & \text { if } f\left(\mathrm{x}_{\mathrm{i}}(t+1)\right) \leq p_{b_{i}}(t)\end{cases}$ updating rule. Clerc [67] proposed constriction factor $(\Psi)$ for ensuring convergence of optimal solution and control of the magnitude of particle velocity. This constriction model also helpful to value selection of $w, c_{1}$ and $c_{2}$. This proposed constriction model with inertia weight is (34) and the pseudo code of PSO algorithm is given in Table 2.

$v_{i}(t+1)=\Psi\left(w \times v_{i}(t)+c_{1} \times \operatorname{rand}_{1}(t) \times\left(p_{b_{i}}(t)-x_{i}(t)\right)+c_{2} \times \operatorname{rand}_{2}(t) \times\left(g_{b}(t)-x_{i}(t)\right)\right)$

where $f: \Re^{\mathrm{m}} \rightarrow \mathfrak{R}$. The global best location $G_{b}$ is calculated by taking the conditions of all particles in the preceding stages is defined as:

$g_{b}(t+1)=\arg \min _{x_{i}} f\left(\left(p_{b_{i}}(t+1)\right)\right)$

$v_{i}$ is the velocity of particle $i ; x_{i}$ denotes the current location of particle $i$ with objective value fitness; $P_{b_{i}}$ is the best past position of particle $i$ itself; $g_{b_{i}}$ is the global best location among the group. $\operatorname{rand}_{1}(t)$, rand $_{1}(t) \in(0,1)$ are the homogeneously distributed random numbers. $c_{1}$ and $c_{2}$ are the positive accelerated constraints. The constants $c_{1}$ and $c_{2}$ express the confidence of the particle has in itself and in its neighbor and these parameters improve fast convergence of the optimal solution. The selection of values of $c_{1}$ and $c_{2}$ are depends on the problem, in the first version of PSO $c_{1}=c_{2}=2$, for better performance on real time problems recent work propose that $c_{1}+c_{2} \leq 4$.

In velocity updating rule (30) has three components, in the first term $v_{i}(t)$ is termed as an inertia component that gives a memory of the immediate past direction. The second term $c_{1} \times \operatorname{rand}_{1}(t) \times\left(p_{b_{i}}(t)-x_{i}(t)\right)$ is termed as a cognitive component that measures the
Table 2 Pseudo code for a PSO algorithm

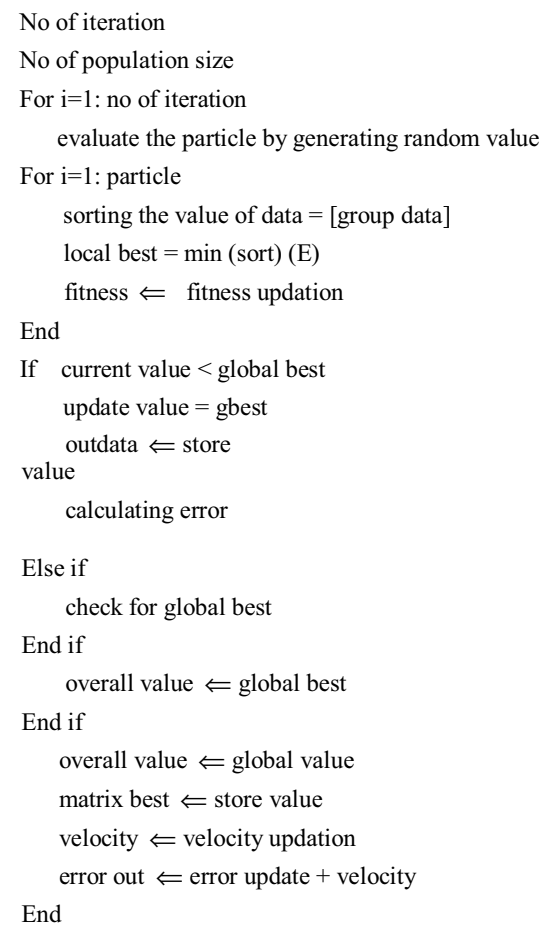


where

$\Psi=\frac{2}{2\left|2-\phi-\sqrt{\phi^{2}-4 \phi}\right|}$

$\phi=c_{1}+c_{2}, \phi>4$

\section{Tuning of FOPID controller for a single conical tank system using PSO algorithm with reference model}

The proposed FOPID controller based PSO algorithm with reference is used to control the level of a single conical tank system model. The detailed proposed block diagram of single conical tank system as exposed in Fig. 6. The referenced model with PID is known as a slave process and plant with FOPID controller based PSO algorithm is known as a master process. In variation between the response from the reference model and plant model is taken as an error which is used for objective function of PSO algorithm. The obtained difference of model error dynamically the correct the FOPID controller parameters in the master process using PSO algorithm. Reference model can be modeled as the expected response from the plant model. Usually, reference model formulation is one of the difficult tasks and it requires a lot of experience about the dynamics of a plant. Simply, for a level process, an overshoot $\left(M_{p}\right)$ is $2 \%$ and settling time $\left(t_{s}\right)$ is 3 min considered for developing reference model.

$\xi=\frac{\ln (M p / 100)}{-\pi} \sqrt{\frac{1}{1+\left[\frac{\ln (M p / 100)}{-\pi}\right]^{2}}}$ and $\omega_{n}=4 / \xi \mathrm{t}_{\mathrm{s}}$

where $\xi$ is the damping ratio, and $\omega_{n}$ is the natural frequency of a system. Thus the transfer function of a reference model is specified in (38)

$G_{R}=\frac{3.55}{s^{2}+2.66 s+3.55}$

Specifying the multi-objective function in the metaheuristic algorithm is one of a way of improving the optimal performance of algorithm [68] and the following objective function (39) is proposed for tuning FOPID controller.

$J\left(K_{p}, K_{i}, K_{d}, \lambda, \mu\right)=\left(\omega_{1} \times \int_{0}^{T}|e(t)|\right)+\left(\omega_{2} \times M_{p}\right)+\left(\omega_{3} \times t_{r}\right)+\left(\omega_{4} \times t_{s}\right)$

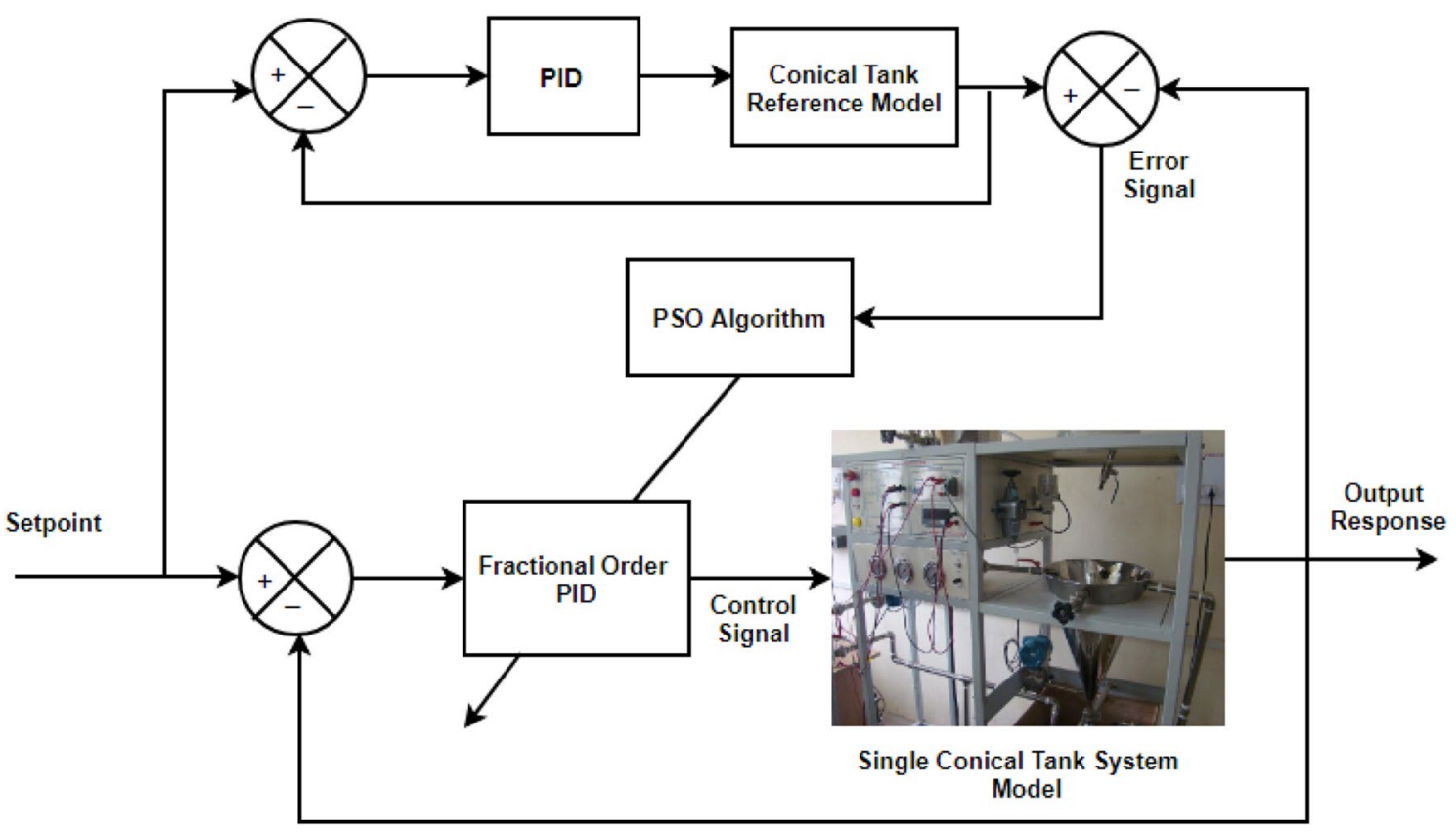

Fig. 6 Proposed block diagram of FOPID controller based PSO with reference model for a single conical tank system 
Table 3 Z-N tuning result of slave PID controller for reference model

\begin{tabular}{llll}
\hline Controller parameters & $K_{p}$ & $K_{i}$ & $K_{d}$ \\
\hline PID & 6.544 & 3.094 & 5.189 \\
\hline
\end{tabular}

Table 4 Values of PSO

\begin{tabular}{ll}
\hline Parameters & PSO \\
\hline Dimension & 5 \\
Number of particles & 50 \\
Number of iteration & 100 \\
$c_{1}$ & 0.8 \\
$c_{2}$ & 1.3 \\
Inertia weight $(w)$ & 0.9 \\
\hline
\end{tabular}

where $e(t)$ is the error (difference between the reference model and real model), $M_{p}$ is maximum peak overshoot, $t_{r}$ is rise time and $t_{s}$ is settling time. The $\omega_{1}, \ldots, \omega_{5}$ are the weighting values of objective function which vary from 0 to 20 in this case. This proposed objective function is applied to the PSO algorithm for optimal tuning of FOPID controller parameters $\left(K_{p}, K_{i}, K_{d}, \lambda, \mu\right)$ for the conical tank system model. It is essential to control the reference model and is done by PID controller with Z-N tuning method.

\section{Simulation results and discussion}

In this segment, the advantage of FOPID controller with reference model is proved through the real time level control of a single conical tank system and compared with PI controller, PID controller and FOPID controller. This work is carried using MATLAB R2013a (Version 8.1.0.604) platform and implemented in a PC with Intel
Core2 Duo Processor with $2.27 \mathrm{GHz}$ speed and $2.00 \mathrm{~GB}$ RAM. National Instruments conical tank system module is used for real time analysis of the proposed controller. The Simulink program is directly interfaced with the real time process system through DAQ module. It is enabled with National instruments VISA serial communication interface. The module supports ASCII data format with a sampling time of $0.1 \mathrm{~s}$ and a baud rate of 38400 . With this, monitoring and control of the real-time process can be easily established with MATLAB software.

The proposed controller consists of two loops: the master process loop consists of FOPID controller with real time conical plant setup and slave process consists of reference model and slave PID controller. The PSO algorithm is utilized for tuning the controller in the master loop and $\mathrm{Z}-\mathrm{N}$ method is used of tuning PID controller in the slave process loop. The $\mathrm{Z}-\mathrm{N}$ tuning result of slave PID controller for reference model as given in Table 3. For optimal tuning of FOPID controller using PSO algorithm, the FOPID parameters ranges are selected as $K_{p} \in[0,40], K_{i} \in[0,10]$, $K_{d} \in[0,10], \lambda \in[0,2], \mu \in[0,2]$ and the parameters of PSO algorithm is listed in Table 4. In order to improve the accuracy and optimum result, PSO algorithm has been run for 20 separate times and best, worst among 20 iterations values listed in Table 5. The minimum value of objective function is denoted as best value and maximum value of objective function value is denoted as worst value among the 20 separate trials and mean of this 20 individual trials is denoted as mean value in Table 5 . This small variation between the best and worst value is due to minor change in the current and reference error ratio at each separate trail. In standard deviation (SD) values are less than one which means that PSO algorithm is performed better in tuning of controller parameters. For tuning controller parameters, multi-object function is proposed that also help full to produced fairly optimized results. The inclusion of time domain
Table 5 Optimized controller parameters using PSO algorithm

\begin{tabular}{llllllll}
\hline Controller & & $K_{p}$ & $K_{i}$ & $K_{d}$ & $\lambda$ & $\mu$ & $J$ \\
\hline PI & Best & 29.136 & 1.898 & - & - & - & 0.9955 \\
& Worst & 29.152 & 1.861 & - & - & - & 0.9985 \\
& Mean & 29.121 & 1.826 & - & - & - & 0.9923 \\
& SD & 00.023 & 0.048 & - & - & - & 0.0026 \\
PID & Best & 28.655 & 4.965 & 8.944 & - & - & 0.9165 \\
& Worst & 28.685 & 4.956 & 8.956 & - & - & 0.9189 \\
& Mean & 28.625 & 4.925 & 8.925 & - & - & 0.9145 \\
& SD & 00.056 & 0.066 & 0.056 & - & - & 0.0016 \\
& Best & 24.852 & 3.466 & 6.473 & 0.469 & 0.656 & 0.7923 \\
& Worst & 24.865 & 3.468 & 6.486 & 0.459 & 0.668 & 0.7985 \\
& Mean & 24.898 & 3.462 & 6.476 & 0.486 & 0.685 & 0.7953 \\
& SD & 00.045 & 0.012 & 0.028 & 0.056 & 0.049 & 0.0023 \\
\hline
\end{tabular}




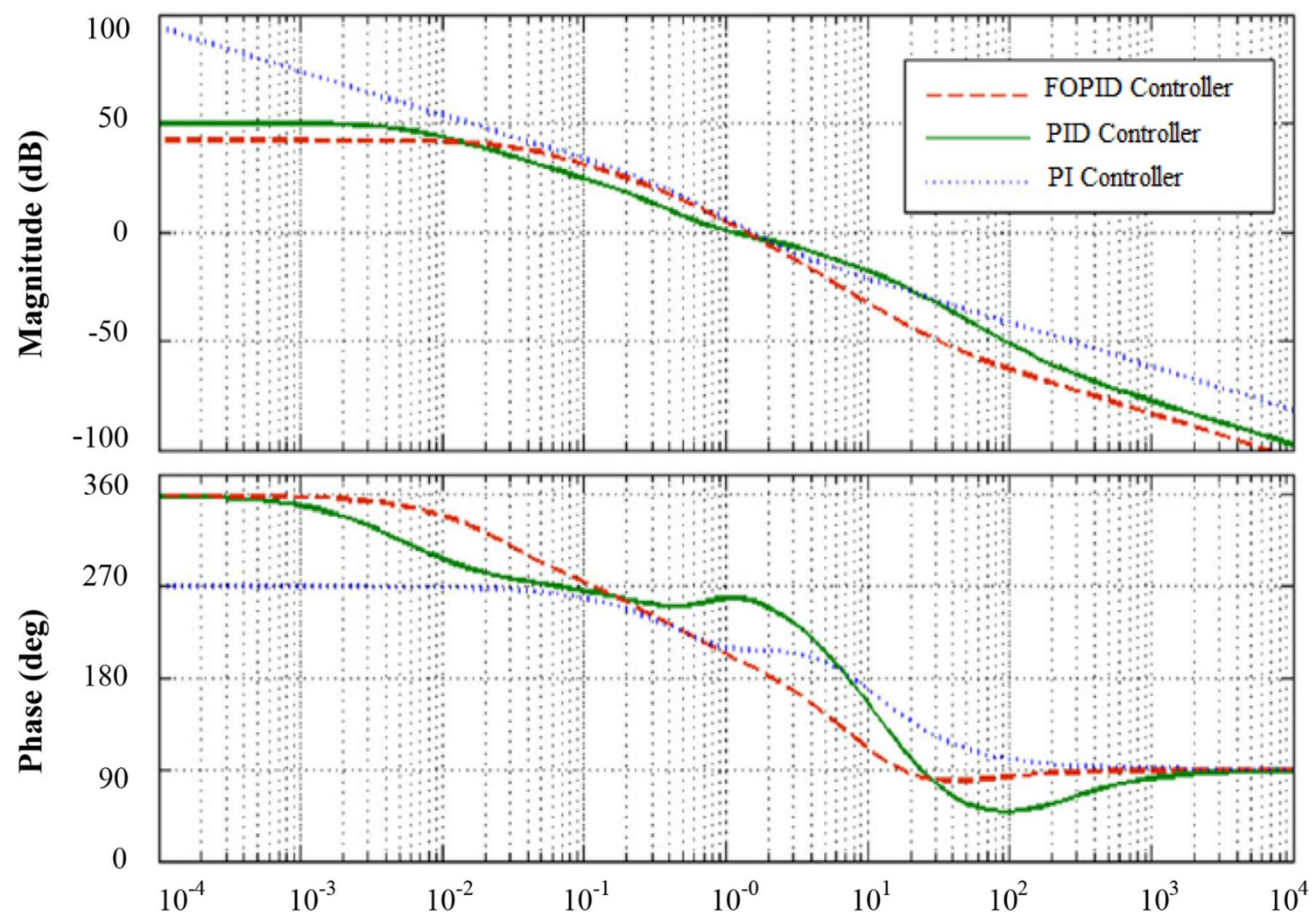

Frequency ( $\mathrm{rad} / \mathrm{sec})$

Fig. 7 Bode diagram of a single conical tank system model

specifications in the multi-objective function is ensures the desired response in the output of the plant.

The controller design is usually based on the approximation of the system to be controlled. In real time, the system behaves differentially than the model indicates. Hence analyzing the robust stability of the system is another important part of the controller design. Figure 7 dissipates the open loop bode plot of FOPID, PID and PI controller. From this plot can be observed that the both magnitude and phase plots are flat and smooth compared to the PID and PI controller and it ensures robustness of system. Moreover, closed loop system with controller can be stable only if the condition,

$|T(j \omega)|\left|G_{\Delta}(j \omega)\right|<1, \forall \omega$

is satisfied [69]. Where

$T(s)=\frac{G_{m}(s) C(s)}{1+G_{m}(s) C(s)}$

and

$G_{\Lambda}(s)=\frac{G_{m 1}(s)-G_{m}(s)}{G_{m}(s)}$
Table 6 Performance analysis of different control methodologies of single conical tank system

\begin{tabular}{lrllr}
\hline Controller & ISE & $\mathrm{M}_{\mathrm{p}}$ & $t_{r}(\mathrm{~s})$ & \multicolumn{1}{c}{$t_{s}(\mathrm{~s})$} \\
\hline PI & 196 & 5.35 & 42.82 & 146.86 \\
PID & 102 & 4.86 & 36.40 & 86.85 \\
FOPID & 75 & 1.34 & 21.36 & 29.34 \\
FOPID with refer- & 41 & 0.93 & 16.79 & 19.45 \\
ence model & & & & \\
\hline
\end{tabular}

$G_{m}(s)$ is the model of the process, $G_{m 1}(s)$ is the model of the process at different operating condition and $C(s)$ is the controller transfer function. The maximum value of $|T(j \omega)|\left|G_{\Delta}(j \omega)\right|$ is found to be 0.658 , hence closed loop system is robust stable. Therefore it can be conclude that reference model with FOPID controller produced better performance compared to the integer order controller.

To analysis the effectives of the proposed controller varying setpoint is given to the input of the controller. Figure 8 shows the performance comparison of different controller methodologies such as PID, FOPI controller, FOPID controller and FOPID controller with reference model and the corresponding numerical data presented in Table 6 . This 


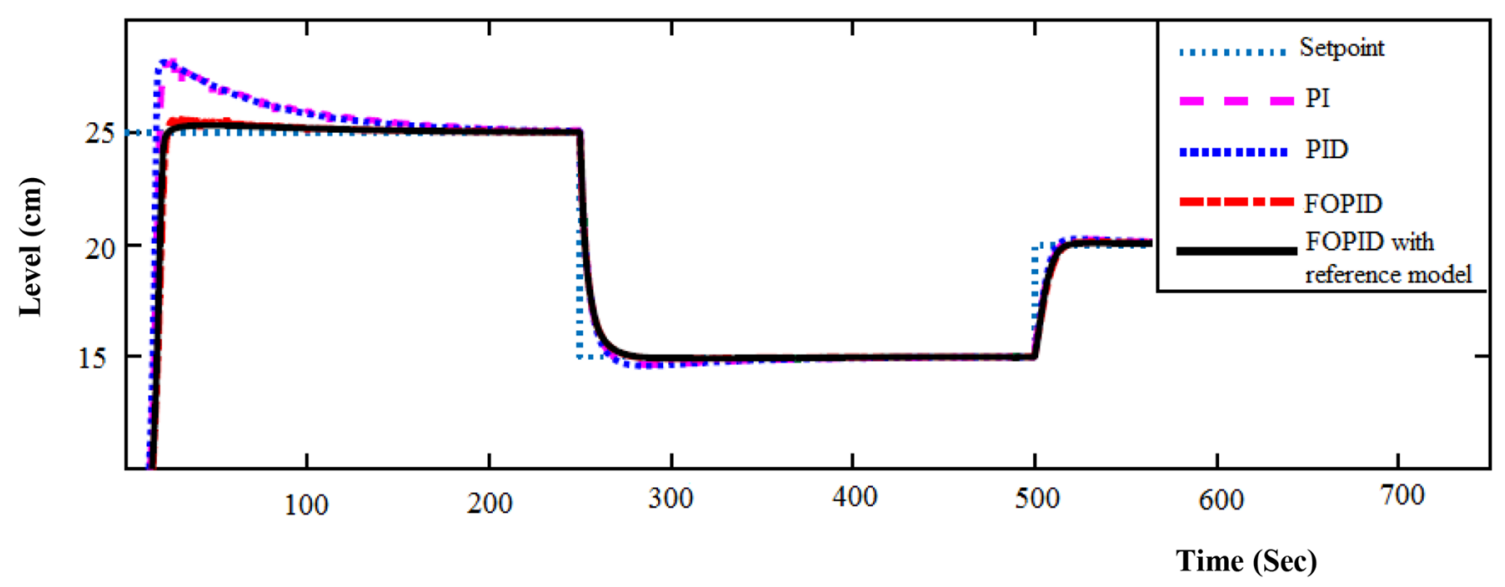

Fig. 8 Comparison of different controller performance of single conical tank system

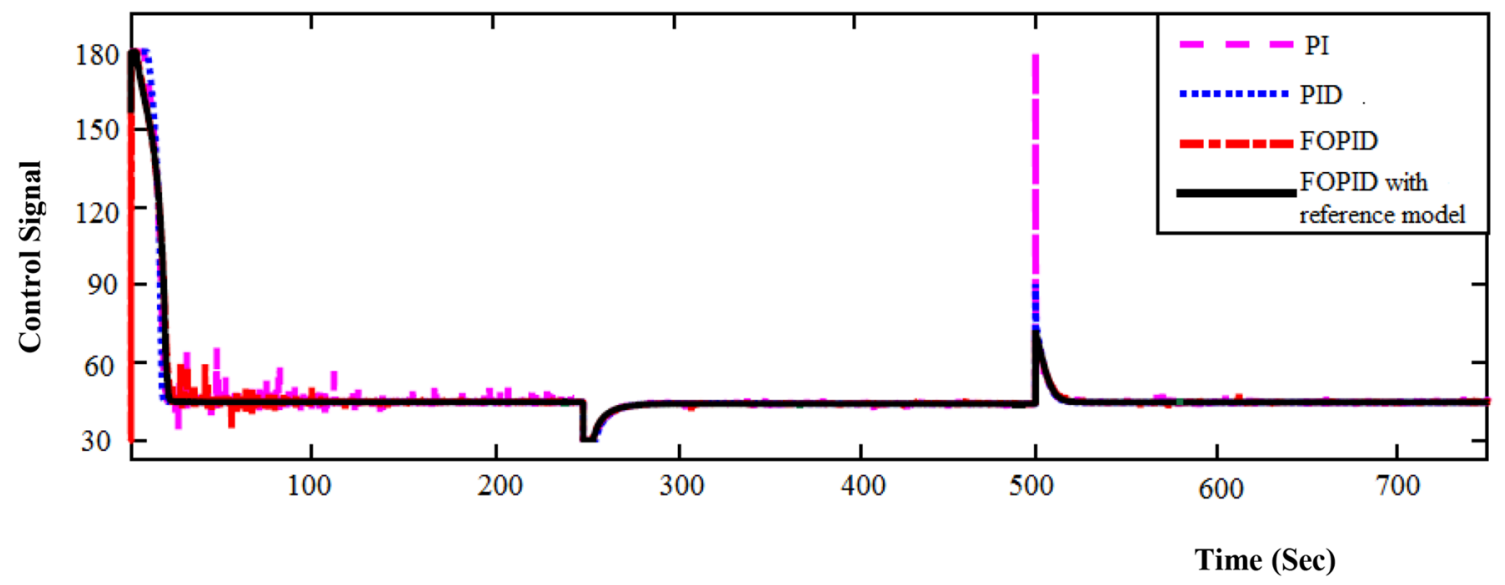

Fig. 9 Control input to single conical tank system

clearly shows the FOPID controller with reference model is produced better performance compared to other controllers. Figure 8 illustrates the control signal of different methodologies which shows FOPID controller with reference model produced smooth response compared to other controllers that avoids damage of sensitive components in the plant. While reducing error difference between slave and master process when time tends to infinity, the trajectory tracking ability of the controller is increased and performance criteria such as selling time, overshoot, rise time, ISE is also improved (Fig. 9).

\section{Conclusion}

The novel FOPID controller with the reference model has been offered for a single conical tank system in this article. A mathematical model of a single conical tank has been identified and approximated as a first order plus dead time model. Closed loop reference model with PID controller is proposed with FOPID controller to tune the highly nonlinear single conical tank model effectively. The PSO algorithm with multi-objective function is used for dynamically adjust the FOPID controller parameters depends on the error difference between the reference model and plant model. The effectiveness of proposed dual loop controller over other controllers are proved in real time under change in setpoint. This critical analysis of the obtained result reveals that the proposed FOPID controller based PSO algorithm with reference model is provided smooth control signal, improved stability and better qualitative performance compared to integer order controllers. Robustness and stability of the controller also analyzed for a single conical tank system model. We believe that the proposed methodology for control can benefit for control practitioners in lot of ways. One 
of the important benefit and key point of this proposed controller is that we can able to obtained required output response by formulating reference model. This work will serve as a new control scheme in process control and several other applications.

\section{Compliance with ethical standards}

Conflict of interest The authors declare that they have no conflict of interests regarding publications of this article.

\section{References}

1. Leibniz GW (1962) Letter from Hanover, Germany, 30 Sept. 1695 to GAV 2. L'Hospital Lebnizen Mathematische Schriften., Olms Verl., Hildesheim. 1962, 301-302

2. Podlubny I, Dorcak L, Kostial I. On fractional derivatives, fractional-order dynamic systems and $\mathrm{Pl} / \mathrm{sup} /$ spl lambda//D/sup/ spl mu//-controllers. In: Decision and control, 1997., Proceedings of the 36th IEEE conference on 1997 Dec 10 (vol. 5, pp 4985-4990). IEEE

3. Roy P, Roy BK (2016) Dual mode adaptive fractional order PI controller with feedforward controller based on variable parameter model for quadruple tank process. ISA Trans 1(63):365-376

4. Ranganayakulu R, Babu GU, Rao AS, Patle DS (2016) A comparative study of fractional order PIX/PIXD $\mu$ tuning rules for stable first order plus time delay processes. Resour-Effic Technol 1(2):S136-S152

5. Sharma R, Rana KP, Kumar V (2014) Performance analysis of fractional order fuzzy PID controllers applied to a robotic manipulator. Expert Syst Appl 41(9):4274-4289

6. Azarmi R, Tavakoli-Kakhki M, Sedigh AK, Fatehi A (2015) Analytical design of fractional order PID controllers based on the fractional set-point weighted structure: case study in twin rotor helicopter. Mechatronics 1(31):222-233

7. Tavazoei MS, Tavakoli-Kakhki M (2014) Compensation by fractional-order phase-lead/lag compensators. IET Control Theory Appl 8(5):319-329

8. Monje CA, Calderón AJ, Vinagre BM, Feliu V (2004) The fractional order lead compensator. In: Computational cybernetics, 2004. ICCC 2004. Second IEEE international conference on 2004 Aug 30. IEEE, pp 347-352

9. Binazadeh T, Shafiei MH (2013) Output tracking of uncertain fractional-order nonlinear systems via a novel fractional-order sliding mode approach. Mechatronics 23(7):888-892

10. Tang Y, Zhang X, Zhang D, Zhao G, Guan X (2013) Fractional order sliding mode controller design for antilock braking systems. Neurocomputing 2(111):122-130

11. Hajiloo A, Nariman-Zadeh N, Moeini A (2012) Pareto optimal robust design of fractional-order PID controllers for systems with probabilistic uncertainties. Mechatronics 22(6):788-801

12. Padula F, Visioli A (2011) Tuning rules for optimal PID and fractional-order PID controllers. J Process Control 21(1):69-81

13. Victor S, Malti R, Garnier H, Oustaloup A (2013) Parameter and differentiation order estimation in fractional models. Automatica 49(4):926-935

14. Tavakoli-Kakhki M, Haeri M (2011) Fractional order model reduction approach based on retention of the dominant dynamics: application in IMC based tuning of FOPI and FOPID controllers. ISA Trans 50(3):432-442
15. Saxena S, Hote YV (2017) Design and validation of fractionalorder control scheme for DC servomotor via internal model control approach. IETE Tech Rev 1:1-2

16. Li H, Luo Y, Chen Y (2010) A fractional order proportional and derivative (FOPD) motion controller: tuning rule and experiments. IEEE Trans Control Syst Technol 18(2):516-520

17. Luo Y, Chen Y (2012) Stabilizing and robust fractional order PI controller synthesis for first order plus time delay systems. Automatica 48(9):2159-2167

18. Padula F, Visioli A (2013) Set-point weight tuning rules for fractional-order PID controllers. Asian J Control 15(3):678-690

19. Tavazoei MS (2015) Reduction of oscillations via fractional order pre-filtering. Sig Process 28(107):407-414

20. Petras I, Hypiusova M (2000) Design of fractional-order controllers via $\mathrm{H}_{\infty}$ norm minimisation. IFAC Proc Vol 33(13):445-448

21. Pan I, Das S, Gupta A (2011) Tuning of an optimal fuzzy PID controller with stochastic algorithms for networked control systems with random time delay. ISA Trans 50(1):28-36

22. Özdemir MT, Öztürk D, Eke Ī, Çelik V, Lee KY (2015) Tuning of optimal classical and fractional order PID parameters forautomatic generation control based on the bacterial swarm optimization. IFAC-PapersOnLine 48(30):501-506

23. Özdemir MT, Öztürk D, Eke İ, Çelik V, Lee KY (2015) Tuning of optimal classical and fractional order PID parameters for automatic generation control based on the bacterial swarm optimization. IFAC-PapersOnLine 48(30):501-506

24. Das S, Pan I, Das S, Gupta A (2012) A novel fractional order fuzzy PID controller and its optimal time domain tuning based on integral performance indices. Eng Appl Artif Intell 25(2):430-442

25. Moradi M (2014) A genetic-multivariable fractional order PID control to multi-input multi-output processes. J Process Control 24(4):336-343

26. Altintas G, Aydin Y (2017) Optimization of fractional and integer order PID parameters using big bang big crunch and genetic algorithms for a MAGLEV system. IFAC-PapersOnLine 50(1):4881-4886

27. Aboelela MA, Ahmed MF, Dorrah HT (2012) Design of aerospace control systems using fractional PID controller. J Adv Res 3(3):225-232

28. Arya Y, Kumar N (2017) BFOA-scaled fractional order fuzzy PID controller applied to AGC of multi-area multi-source electric power generating systems. Swarm Evol Comput 28(32):202-218

29. Rajasekhar A, Jatoth RK, Abraham A (2014) Design of intelligent $\mathrm{PID} / \mathrm{PI} \lambda \mathrm{D} \mu$ speed controller for chopper fed DC motor drive using opposition based artificial bee colony algorithm. Eng Appl Artif Intell 31(29):13-32

30. Kumar A, Kumar V (2017) A novel interval type-2 fractional order fuzzy PID controller: design, performance evaluation, and its optimal time domain tuning. ISA Trans 31(68):251-275

31. Alagoz BB, Ates A, Yeroglu C (2013) Auto-tuning of PID controller according to fractional-order reference model approximation for DC rotor control. Mechatronics 23(7):789-797

32. Sánchez HS, Padula F, Visioli A, Vilanova R (2017) Tuning rules for robust FOPID controllers based on multi-objective optimization with FOPDT models. ISA Trans 31(66):344-361

33. Sharma R, Gaur P, Mittal AP (2015) Performance analysis of twodegree of freedom fractional order PID controllers for robotic manipulator with payload. ISA Trans 30(58):279-291

34. Sikander A, Thakur P, Bansal RC, Rajasekar S (2017) A novel technique to design cuckoo search based FOPID controller for AVR in power systems. Comput Electr Eng

35. Xu Y, Zhou J, Xue X, Fu W, Zhu W, Li C (2016) An adaptively fast fuzzy fractional order PID control for pumped storage hydro unit using improved gravitational search algorithm. Energy Convers Manag 1(111):67-78 
36. Biswas A, Das S, Abraham A, Dasgupta S (2009) Design of fractional-order PI $\lambda D \mu$ controllers with an improved differential evolution. Eng Appl Artif Intell 22(2):343-350

37. Tang Y, Cui M, Hua C, Li L, Yang Y (2012) Optimum design of fractional order PI $\lambda$ D $\mu$ controller for AVR system using chaotic ant swarm. Expert Syst Appl 39(8):6887-6896

38. Etedali S, Zamani AA, Tavakoli S (2018) A GBMO-based PI $\lambda$ D $\mu$ controller for vibration mitigation of seismic-excited structures. Autom Constr 31(87):1-2

39. Chaib L, Choucha A, Arif S (2015) Optimal design and tuning of novel fractional order PID power system stabilizer using a new metaheuristic Bat algorithm. Ain Shams Eng J

40. Ateş A, Yeroglu C (2016) Optimal fractional order PID design via Tabu Search based algorithm. ISA Trans 31(60):109-118

41. Rajesh R, Deepa SN (2019) Design of direct MRAC augmented with 2 DoF PIDD controller: an application to speed control of a servo plant. J King Saud Univ-Eng Sci

42. Barbosa RS, Machado JT, Ferreira IM (2004) Tuning of PID controllers based on Bode's ideal transfer function. Nonlinear Dyn 38(1-4):305-321

43. Li X, Wang Y, Li N, Han M, Tang Y, Liu F (2017) Optimal fractional order PID controller design for automatic voltage regulator system based on reference model using particle swarm optimization. Int J Mach Learn Cybern 8(5):1595-1605

44. Ahmad W, Htut MM (2009) Neural-tuned PID controller for pointto-point (PTP) positioning system: model reference approach. In: 2009 5th International colloquium on signal processing and its applications 2009 Mar 6. IEEE, pp 140-144

45. Wang W, Nonami K, Ohira Y (2008) Model reference sliding mode control of small helicopter XRB based on vision. Int J Adv Rob Syst 5(3):26

46. Jeng JC (2018) Data-based tuning of PID controllers: a combined model-reference and VRFT method. In: PID control for industrial processes. IntechOpen

47. Ramanathan P, Mangla KK, Satpathy S (2018) Smart controller for conical tank system using reinforcement learning algorithm. Measurement 1(116):422-428

48. Vijayalakshmi S, Manamalli D, PalaniKumar G (2014) Closed loop experimental validation of linear parameter varying model with adaptive PI controller for conical tank system. J Control Eng Appl Inform 16(4):12-19

49. Ravi VR, Thyagarajan T (2014) Adaptive decentralized PI controller for two conical tank interacting level system. Arab J Sci Eng 39(12):8433-8451

50. Tamilselvan GM, Aarthy P (2017) Online tuning of fuzzy logic controller using Kalman algorithm for conical tank system. J Appl Res Technol 15(5):492-503

51. Travieso-Torres JC, Duarte-Mermoud MA, Beytía-Cancino O (2017) Taylor polynomial approximation and adaptive passivitybased control applied to the level regulation of a conical tank. Asian J Control 19(5):1722-1730

52. Machado JT, Kiryakova V, Mainardi F (2011) Recent history of fractional calculus. Commun Nonlinear Sci Numer Simul 16(3):1140-1153

53. Yeroglu C, Tan N (2011) Note on fractional-order proportionalintegral-differential controller design. IET Control Theory Appl 5(17):1978-1989
54. Shah P, Agashe S (2016) Review of fractional PID controller. Mechatronics 30(38):29-41

55. Oldham K, Spanier J (1974) The fractional calculus theory and applications of differentiation and integration to arbitrary order. Elsevier, Amsterdam

56. Polubny I (1999) Fractional-order systems and PI $\lambda D \mu$ controller. IEEE Trans Autom Control 44:208-214

57. Podlubny I (1998) Fractional differential equations: an introduction to fractional derivatives, fractional differential equations, to methods of their solution and some of their applications. Academic Press, London

58. Ortigueira MD, Trujillo JJ (2009) Generalized GL fractional derivative and its Laplace and Fourier transform. In: Proceedings of the ASME 2009 international design engineering technical conferences computers and information in engineering conference, IDETC/CIE 2009

59. Oustaloup A, Levron F, Mathieu B, Nanot FM (2000) Frequencyband complex noninteger differentiator: characterization and synthesis. IEEE Trans Circuits Syst I: Fundam Theory Appl 47(1):25-39

60. Oustaloup A, Melchior P, Lanusse P, Cois O, Dancla F (2000) The CRONE toolbox for Matlab. In: Computer-aided control system design, 2000. CACSD 2000. IEEE international symposium on 2000. IEEE, pp 190-195

61. Ziegler JG, Nichols NB (1942) Optimum settings for automatic controllers. trans. ASME 64(11)

62. Cheng GS, Hung JC (1985) A least-squares based self-tuning of PID controller. In: Proceedings of the IEEE South East Conference, Raleigh, North Carolina, USA, pp 325-332

63. Prabhu ES, Chidambaram M (1991) Robust control of a distillation column by the method of inequalities. J Process Control 1(3):171-176

64. Sundaresan KR, Krishnaswamy PR (1978) Estimation of time delay time constant parameters in time, frequency, and Laplace domains. Can J Chem Eng 56(2):257-262

65. Eberhart R, Kennedy J (1995) A new optimizer using particle swarm theory. In: Micro machine and human science, 1995. MHS'95., Proceedings of the sixth international symposium on 1995 Oct 4, IEEE, pp 39-43

66. Shi Y, Eberhart R (1998) A modified particle swarm optimizer. In: Evolutionary computation proceedings, 1998. IEEE World Congress on computational intelligence, the 1998 IEEE international conference on 1998 May 4, IEEE, pp 69-73

67. Clerc M (1999) The swarm and the queen: towards a deterministic and adaptive particle swarm optimization. In: Evolutionary computation, 1999. CEC 99. Proceedings of the 1999 Congress on 1999 (vol 3, pp 1951-1957). IEEE

68. Zamani M, Sadati N, Ghartemani MK (2009) Design of an $\mathrm{H}_{\infty}$ PID controller using particle swarm optimization. Int J Control Autom Syst 7(2):273-280

69. Morari M, Zafiriou E (1989) Robust process control. Prentice Hall, Englewood cliffs

Publisher's Note Springer Nature remains neutral with regard to jurisdictional claims in published maps and institutional affiliations. 